\title{
Locomotion methods of microrobots and targeted drug delivery
}

\author{
Alper $\operatorname{Kurt}^{1}{ }^{(\mathbb{D}}$, Recep Tayyip Özbek ${ }^{2} \mathbb{D}$ \\ ${ }^{1}$ Department of Electrical and Electronics Engineering, Özyeğin University, Istanbul, Turkey \\ ${ }^{2}$ Kocaeli University, Department of Mechanical Engineering, Kocaeli, Turkey
}

\begin{abstract}
Many of the drugs used in the treatment of diseases, such as cancer, spread throughout the body, causing tissue and healthy cell loss. Patients taking these medications may experience serious side effects. To overcome these problems, scientists have developed many types of microrobots and operating principles which can release drugs manageably and locally and can be remotely controlled with external sources. In this review, four different movement types that we selected from the movement mechanisms of microrobots are examined in detail. These motion types are magnetic motion, acoustic motion, microorganism-based motion, and chemical reaction-based motion, respectively. In this review, we discuss how each movement mechanism works and what kind of experimental studies it has today. In addition, the shortcomings of these movement mechanisms and the aspects that need to be improved are mentioned. In general, this review sheds light into how microrobots facilitate human health with targeted drug delivery technology and forthcoming biomedical studies.

Keywords: Microrobots, mobile robot, targeted drug delivery.
\end{abstract}

Feyman $^{[1]}$ talked about miniature machines in his conference on "There's Plenty of Room at the Bottom" in 1959, and the basic idea of microrobots was suggested for the first time. In the science fiction movie Fantastic Voyage, released in 1966, the miniaturized submarine crew injected into the human body and performed surgeries, and this increased the popularity of the concept of microrobotics.

Microrobots are microscopic machines that have partially or completely self-contained capabilities such as movement, sensing and learning, re-programmable, with a maximum size of $1 \mathrm{~mm}$ and minimum $0.1 \mu \mathrm{m} \cdot{ }^{[2,3]}$ In the past decade, microrobotics has emerged as a new robotic field for creating small untethered robots that can access and work actively in small areas that are dangerous or difficult to reach. ${ }^{[4,5]}$

In traditional methods used for disease therapies, medicaments are usually taken into the body orally or by injection. Thus, the taken medicaments penetrate a large part of the body. This condition causes damage to body cells and tissues. At the same time, it reveals serious health problems and unwanted side effects for the patient. ${ }^{[6]}$

According to the data of interviews with 441 patients obtained from 12 separate cancer treatment centers in New South Wales, Australia, $86 \%$ of the patients who received chemotherapy treatment stated that they had at least one side effect. However, $67 \%$ of the patients stated that they had six or more side effects. ${ }^{[7]}$ Most of the patients who received systemic treatment had many side effects, particularly fatigue, pain and intestinal problems. ${ }^{[7]}$

Microrobots allow to reach internal body parts such as the nervous system that are difficult to reach ${ }^{[8]}$ and deliver drugs to targeted areas by autonomous or remote control. In addition, drugs cannot provide restoration of tissue or organ functions alone, but successfully complete 
the restoration by reaching the desired areas of damaged tissues and organs. It also offers opportunities for cargo protection, environmental perception, monitoring and improvement, as well as the ability to reduce the side effects of drugs taken directly and regulate drug intake..$^{[9,10]}$ Regarding these features, targeted drug delivery with untethered microrobots is a very good solution for these problems. ${ }^{[6]}$

\section{LOCOMOTION IN MICROROBOTS}

Microrobots can perform operations such as therapy to the targeted area, minimally invasive surgery, and sensitive cell or drug delivery in the viscous environment of biological systems. ${ }^{[1]}$ In addition, safe robot-human interactions are ideal tools for disease diagnosis and treatment. ${ }^{[12,13]}$

Reynolds (Re) number is important when considering movement in microrobots. The main keys here are density, fluid velocity, pipe diameter and dynamic viscosity of the fluid. When the Reynolds number is much less than 1 ( $R e \quad<<1)$ inertia forces are negligible besides viscosity forces. In this case, the movements of microrobots in a fluid can be compared to a human swimming in a pool of honey or jam. ${ }^{[14]}$ The high drag forces created by the viscous environment that limit the movements of microrobots is a problem that needs to be solved and considered. Researchers who want to overcome these problems have developed various methods to locate and direct in vivo microrobots to their targets. Some of these methods are magnetically-driven, motile microorganisms-driven, acoustic-aided, and chemical reaction-based locomotion..$^{[15]}$

\section{MAGNETICALLY-DRIVEN LOCOMOTION}

The magnetic field for drug transportation was first used in the 1950s. ${ }^{[16,17]}$ Meyers et al. $^{[18]}$ in 1963 developed a new method by placing a horseshoe magnet outside the body and collecting small iron particles in a specific area of the body. In 1996, for the first time in clinical studies, magnetic drug transport with a permanent magnet with a magnetic flux density of $0.8 \mathrm{~T}$ placed close to the surface of the body skin was used for tumor treatment. ${ }^{[19]}$ Since then, drug targeting has found a wide scope of work and has developed rapidly. ${ }^{[20,21]}$ Magnetic fields have attracted attention due to its insensitivity to biological materials, its ability to position precisely and its ability to operate without direct contact. $^{[14,22]}$

Magnetic fields produced outside the body are a good solution to overcome the problems encountered in in vivo procedures. Using these magnetic fields generated outside the body, microrobots can be remotely controlled and strengthen their spatial maneuverability. Unlike other methods, magnetic fields penetrate biological tissues and additional materials without any damage. Therefore, their use in bioengineering applications is very promising. Magnetic fields that change with time and their spatial gradients provide the basis for the magnetic activation of microrobots. ${ }^{[8,23]}$

Microrobots used for magnetic drug transport contain particles such as ferric oxide that interact with the magnetic field in their structures so that they can be transported to desired areas in the body. ${ }^{[6]}$ Microrobots equipped with these particles feel magnetic force and torque when they settle in the magnetic field. This magnetic force and torque allow microrobots to control their movement and direction in vivo. ${ }^{[24]}$

The locomotion mechanisms of microorganisms observed in nature have been a great source of inspiration in determining the design and motion principles of magnetic microrobots. These designs are generally collected in three different categories as flagellum-like design, helical tail-like design and cilia-like design (Figure 1). ${ }^{[14]}$

Regarding its one-dimensional structure, the simplest design is a flexible flagellum. When connected to a magnetic head, a flexible plate or rod can be magnetically moved. ${ }^{[25,26]}$ First, Dreyfus et al. ${ }^{[27]}$ produced a bendable artificial flagellum using DNA fibers attached to a red blood cell and super-paramagnetic particles attached to one end, and through this flagellum, it provides magnetic mobility to a microrobot.

The first helical microrobot to mimic bacterial movement, the artificial bacterial flagellum (ABF), was first introduced by Bell et al., ${ }^{[28]}$ in 2007 and, then, characterized by Zhang et al., ${ }^{[29]}$ in 2009. The ABF consists of a helical tail attached to a soft-magnetic head. This helical tail is $2.8 \mathrm{~mm}$ in diameter and 30-100 $\mathrm{mm}$ in length. 


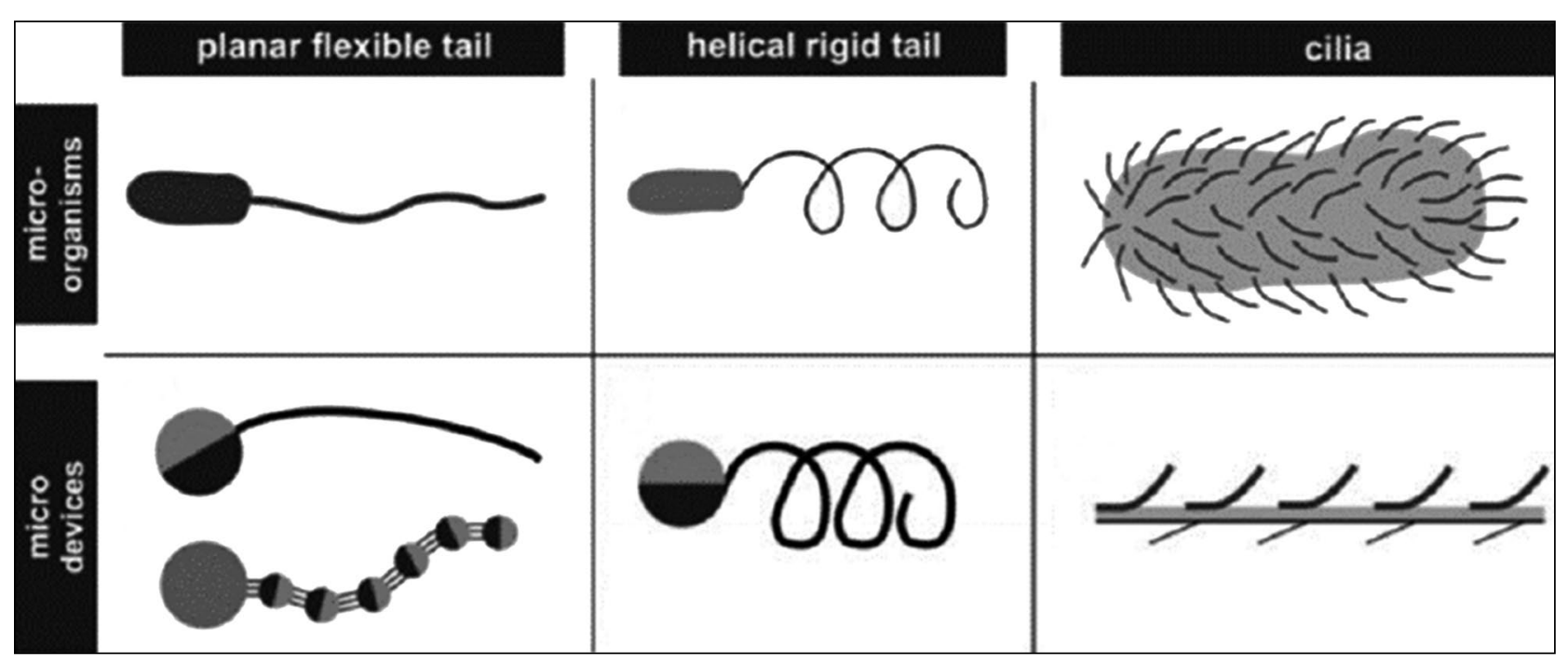

Figure 1. Structural designs inspired by microorganisms to move. ${ }^{[14]}$

Cilia is among the first intensively studied filaments in nature. ${ }^{[30]}$ Cilia usually covers the entire surface and moves in a coordinated manner. If a cilia movement like this is to be applied for Microrobots, independent movement is required for each cilium. ${ }^{[31]}$ However, artificial magnetic cilia move simultaneously. To date, there are no microrobots that use cilia for locomotion. Instead, artificial cilia is applied to a surface to transport the fluid it has. ${ }^{[14]}$

For the safe use of magnetic microrobots in in vivo settings, static magnetic fields below $8 \mathrm{~T}$ should be created. ${ }^{[32]}$ Magnetic fields above this value cause heating of the applied tissues and cause tissue damage. To ensure safety of these studies, it is necessary to optimize the magnetic components of microrobots, according to regulatory guidelines, acceptable magnetic field and gradient exposure levels. ${ }^{[33]}$

\section{ACOUSTIC-GUIDED LOCOMOTION}

Acoustic fields are another way of controlling remote routing in microrobots. Acoustic flux with acoustic radiation force is one of the most beneficial effects of acoustic fields. It is possible to generate an acoustic radiation force with a standing wave generated by reflecting back and forth from the resonator. This radiation force creates a drag force to drive microrobots with sound pressure nodes and anti-nodes, which are points of maximum and minimum amplitude. ${ }^{[34,35]}$
The direction of this motion can be predicted and changed by wave functions corresponding to this motion. As a result, multiple micro-objects can be clustered in desired regions through this method. ${ }^{[34,36]}$

Since the absence of selectivity in acoustic areas, it is ineffective to approach it individually. However, the recently introduced acoustic radiation force applied in an oscillating manner can provide selective addressability. In this system, vibrating bubbles are squeezed into the micro-swim bodies to create sufficient propulsion for movement. It is possible to operate this system by selecting a single micro-swimmer in a group of micro-swimmers with different bubble sizes (Figure 2). ${ }^{[37,38]}$

The movement of these micro robots is provided by the acoustic radiation force created by the acoustic fields, while the direction of the movement is performed by the effect of the magnetic field. This change of direction is achieved as a result of the $20 \mathrm{~nm}$ magnetic nickel (Ni) coating sprayed on the robots as a result of this coating creating torque and force effects in the magnetic field. ${ }^{[39]}$

\section{MOTILE MICROORGANISMS-DRIVEN LOCOMOTION}

The ability to feel within the human body, to have computational capacity and power for 


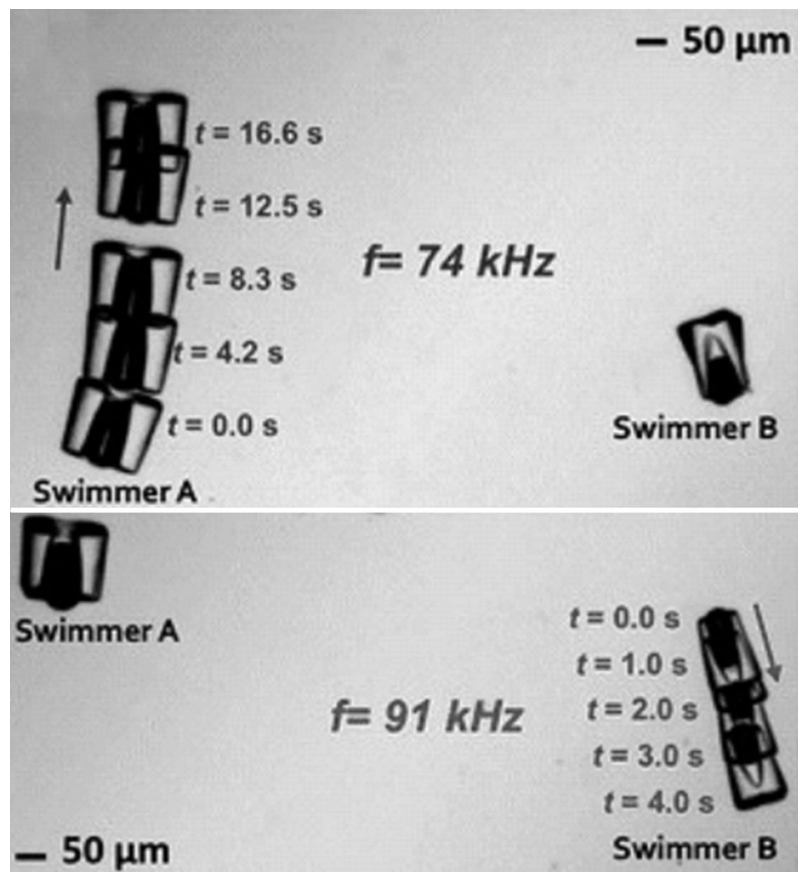

Figure 2. Moving two microrobots one by one with frequency change. ${ }^{[39]}$

movement is the key to a successful microrobotic practice. Therefore, microorganism-based moving microrobots provide a high level of autonomy, as they can sense the changes happening around them and react quickly. Additionally, many microorganisms can travel ten times their body length per second. Therefore, they are used in biohybrid technologies, medical applications and drug transport with microrobots. ${ }^{[40]}$

Microorganisms such as bacteria and spermatozoa can be mobilizers for microrobots. These microorganisms move through in the microscale, using biomechanical energy to move their flagella. Unlike artificial propellants, the movement mechanisms of microorganisms are much more efficient in a low Reynolds number environment. ${ }^{[41,42]}$

The movements of the hybrid microrobots are controlled by the reactions of the microorganisms they use together to physical or chemical stimuli from outside. These movements take place in the desired way is called "taxis". ${ }^{43]}$ Microorganisms can also use "taxis" to control the movements of microrobots, regarding their ability to react instantaneously to small and delicate changes in their surroundings. Chemical, ${ }^{[44]}$ photo $^{[45]}$ and
$\mathrm{pH}^{[46]}$ taxis methods are applied according to the type of microorganisms used.

Kim et al. ${ }^{[4]}$ showed that it was possible to chemically control microparticles driven by bacteria in a microfluidic liquid. The researchers identified the direction of the Serratia Marcescens floating bacteria using the chemical L-Aspartate (chemoattractant) and used this bacterium as a driving force. In this study, the bacteria displayed a clear ability to direct in all test trials involving chemical stimuli. This capability was well-suited for targeted drug delivery.

Weibel et al. ${ }^{[48]}$ showed that manipulation of micro-objects was carried out using phototaxisbased control of algae with flagella. The movement of algae is achieved by turning the LED light on and off in the desired area. Micro objects with a diameter of $3 \mu \mathrm{m}$ adhere to the algae through the surface chemistry and were released from the algae in the desired areas by photochemical effects. Zhuang et al. ${ }^{[49]}$ provided the movement of microrobots repelled by multi-bacteria through the biased behavior toward PH. Thanks to a stable $\mathrm{pH}$ gradient, microrobots are mobilized in a unidirectional and versatile way.

The taxis methods can be used appropriately in short distance systems. For biomedical applications such as targeted drug delivery, microorganisms must be controlled over a long distance. In this context, the magnetic field is a strong candidate for a long-distance remote-control system. ${ }^{.50]}$ Magnetic field is used as a macro management method in regions with high blood flow, such as large blood vessels, while microorganism-based repulsion is used to provide micro-control system in small blood vessels. ${ }^{[51]}$

\section{CHEMICAL REACTION-BASED LOCOMOTION}

The chemical reaction-based movement of microrobots takes place by using the chemical energy around it. When using this chemical energy, it can use organic fuels such as glucose or inorganic fuels such as hydrogen peroxide as the input energy source. Micromotors have been developed that can transform the energy stored in chemical bonds into use and turn them into directional motion. Since inertial forces are negligible in a low Reynolds number environment, 
microrobots that take their mobility from chemical reaction must have a continuous chemical energy conversion and an asymmetric structure. These microrobots often contain an active metal that acts as a catalyst to trigger the chemical reaction and an unreactive material to fabricate an asymmetric design. ${ }^{[8.52]}$

One of the widely used chemical-based mechanism of action is the method of generating a self-phoretic repulsive force induced by regional chemical variables in the microrobot's environment. This repulsion method can only generate enough force to move microrobots smaller than $3 \mu \mathrm{m} .{ }^{[53,54]}$ Paxton et al., ${ }^{[55]}$ discovered the self-phroteric repulsion ability of Au-Pt bimetallic nanorods in hydrogen peroxide first. Later, they confirmed that this repulsive force is generated by autoelectrophoresis, which involves electrochemical dissolution of $\mathrm{H}_{2} \mathrm{O}_{2} \cdot{ }^{[56]}$

Another widely used chemical based action mechanism is based on the bubble rebound mechanism. In this mechanism, the movement is provided by the force generated by the chemical trapped bubbles in a groove spreading out of the opening in the groove. The resultant recoil force is used to move the microrobot, which is usually produced as a tube containing catalytic material on its inner surface.

The tubular design makes it easier for the bubbles to cluster and move out of the groove. As the gas cluster accumulates in the tube increases, the pressure inside increases, so the bubbles are sprayed out from the opening in the tube. Meanwhile, the momentum change directed the movement in the opposite direction. The speed of the microrobot has been adjusted by stimulating the momentum change with stimuli such as temperature, light and ultrasound. ${ }^{[57-59]}$

Since ultrasound affects the bubble release, it can be used to adjust the speed of the moving micromotor by spraying bubbles. In this way, instantaneous changes in the speed of the micromotor can be made, while on-off control is achieved. ${ }^{[60]}$ According to another method, speed control between 100-250 $\mu \mathrm{m}$ per second was achieved by folding and unfolding curled polymer platinum tubes using temperature changes. ${ }^{[61]}$

Coating magnesium particles with a red blood cell membrane has been shown to purify the serum-like solution from protein toxins of its toxic properties. ${ }^{[62]}$ However, local $\mathrm{pH}$ rise due to magnesium peroxide build-up is a potential safety risk for this system. Depending on the particular body area, this may be an acceptable and advantageous means of providing power. For example, micro-robots based on magnesium and zinc powered micromotors can be well suited for operations in the acidic gastrointestinal tract. For this purpose, the autonomous action of the zincbased micromotor in the stomach of a live mouse was first applied in vivo. ${ }^{[63]}$

A tubular catalytic microrobot that can move in $\mathrm{H}_{2} \mathrm{O}_{2}$ solution has been produced. The production of this tubular microrobot was carried out by rolling the multi-layers of $\mathrm{Pt}, \mathrm{Au}, \mathrm{Fe}$, Ti. The inner layer, Pt layer, enables the catalytic reaction, the $\mathrm{Au}$ layer the junction between the layers, the Fe layer magnetic orientation and the Ti layer a controlled rolling process. Pt on the inner surface of the tube acts as a chemical reaction chamber for the $\mathrm{O}_{2}$ gas bubbles formed as a result of the catalytic dissolution of $\mathrm{H}_{2} \mathrm{O}_{2}$. The $\mathrm{H}_{2} \mathrm{O}_{2}$ solution is pumped as fuel from the front end of the tubular microrobot into the chemical reaction chamber, while bubbles of $\mathrm{O}_{2}$ gas are sprayed from the rear end of the microrobot. In this way, the microrobot always gains the ability to move. The direction of movement is adjusted by an externally applied magnetic field. ${ }^{[64]}$ The use of $\mathrm{H}_{2} \mathrm{O}_{2}$ solution as a fuel provides fast and effective mobility. However, high density $\mathrm{H}_{2} \mathrm{O}_{2}$ is biologically incompatible in in vivo applications due to its high oxidation. Therefore, finding new biocompatible fuels for viable in vivo applications is essential. ${ }^{[65]}$

One of the methods recommended for biocompatible microrobot production aims to be able to move in an acidic environment such as the stomach. This movement takes place thanks to the hydrogen bubbles spraying continuously thanks to the redox reactions occurring on the zinc surface on the inner surface of the microrobot. ${ }^{[65]}$ Despite the development of bubble rebound mechanisms using biocompatible fuels, the use of active metals such as zinc shortens the operation time of microrobots. However, the main problem is that the bubbles resulting from the bubble rebound method are undesirable in any area other than the digestive system. ${ }^{\text {[52] }}$ 


\section{DISCUSSION}

Microrobots are in the first place in the direction of being a promising technology branch in terms of delivering drugs to the desired area of traditional medicine or reaching the areas that are desired to be viewed and difficult to reach in 10 years. Scientists contribute to the development of this field by conducting experiments and studies in the laboratory environment or in living organisms every day. Since the production and design of microrobots requires high technology, it is a very difficult field to study. Scientists have developed different production materials according to different production methods and usage areas to overcome this difficulty. It is of utmost importance that microrobots that need to function in very small spaces can be remotely controlled in these areas in a controlled manner. In this context, a lot of work has been done on the investigation and development of the movement mechanisms of microrobots.

In this review, some of the action mechanisms of microrobots are examined in detail. These motion mechanisms are presented in four different categories as magnetic motion, acoustic motion, microorganism-based motion and chemical reaction-based motion. The basic idea in the magnetic motion mechanism is that microrobots equipped with particles that interact with the magnetic field feel a force and torque that create movement on them when exposed to a magnetic field. Since the magnetic field is highly operable in terms of delivering microrobots to the desired area, it has often been used in conjunction with other methods. It has been shown as a biocompatible method due to the low interaction of the magnetic field with cells in the body. However, for this method to remain biocompatible, the magnetic field used must be less than $8 \mathrm{~T}$. Another problem is that magnetic field generating systems used for remote control of microrobots are large and costly. Acoustic movement is a method of moving microrobots by generating acoustic radiation force with the help of waves standing in the body. Since this method is inadequate in terms of selectivity and reaching the desired areas, its use is less preferred than other methods.

In the microorganism-based movement, microorganisms that already have high mobility and the ability to react to changes in the environment are used as the driving motor that moves microrobots. In this way, a controlled movement can be achieved by making changes in the environment where microrobots are located in the body. Since microorganisms can react instantaneously to stimuli around them, it has significantly improved the mobility of microrobots. However, the force created by microorganisms is only suitable for moving robots of certain sizes and can only be used in vessels with low blood flow velocity. Therefore, if drug transport is to be carried out over a long distance, it is more efficient to carry out this transport with a magnetic field and then to perform the movement with the microorganism when the microrobot reaches the desired area. The movement based on chemical reaction uses the energy it generates using organic or inorganic fuels to move. Although it is easy to reach the desired area in this type of movement, it is a biologically hazardous method, as substances that can cause toxicity in the body such as hydrogen peroxide are used. The use of zinc does not cause toxic accumulation, but the use of $\mathrm{O}_{2}$ gas bubbles due to the working mechanism is limited for all parts of the body except the digestive system. To overcome this danger, new fuels that are harmless to the human body are being investigated.

In conclusion, microrobots are promising candidates to save individuals from painful treatments that leave them in hospitals for weeks in the near future. Microrobot studies will allow to eliminate many side effects seen in cancer treatment and affect the whole body and to minimize tissue damage caused by the drug by performing the treatment in a small area. Microrobots in micron or even nanometric sizes will take the health sector to the next level by aiming to reach all parts of the human body. These robots will erase today's most feared and most human-fatal problems such as cancer and cardiovascular diseases from the stage of history and save individuals from painful, long-term and very side-effective treatments.

\section{Declaration of conflicting interests}

The authors declared no conflicts of interest with respect to the authorship and/or publication of this article. 


\section{Funding}

The authors received no financial support for the research and/or authorship of this article.

\section{REFERENCES}

1. Meeker DC, Maslen EH, Ritter RC, Creighton FM. Optimal realization of arbitrary forces in a magnetic stereotaxis system. IEEE Transactions on Magnetics 1996;32:320-8.

2. Diller E, Sitti M. Foundations and Trends in Robotics. In: Foundations and Trends in Human-Computer Interaction; 2013.

3. Sitti M. Microscale and nanoscale robotics systems [Grand Challenges of Robotics]. IEEE Robotics \& Automation Magazine 2007;14:53-60.

4. Sitti M. Mobile Microrobotics. Cambridge: MIT Press; 2017.

5. Zeng H, Wasylczyk P, Wiersma DS, Priimagi A. Light robots: Bridging the gap between microrobotics and photomechanics in soft materials. Adv Mater 2018;30:e1703554.

6. Liu YL, Chen D, Shang P, Yin DC. A review of magnet systems for targeted drug delivery. J Control Release 2019;302:90-104.

7. Pearce A, Haas M, Viney R, Pearson SA, Haywood P, Brown $C$, et al. Incidence and severity of self-reported chemotherapy side effects in routine care: A prospective cohort study. PLoS One 2017;12:e0184360.

8. Ceylan H, Giltinan J, Kozielski K, Sitti M. Mobile microrobots for bioengineering applications. Lab Chip 2017;17:1705-24.

9. Rautioa J, Chikhale PJ. Drug delivery systems for brain tumor therapy. Curr Pharm Des 2004;10:1341-53.

10. Erkoc P, Yasa IC, Ceylan H, Yasa O, Alapan Y, Sitti M. Mobile microrobots for active therapeutic delivery. Advanced Therapeutics 2019;2:1800064.

11. Kim S, Lee S, Lee J, Nelson BJ, Zhang L, Choi H. Fabrication and Manipulation of Ciliary Microrobots with Non-reciprocal Magnetic Actuation. Sci Rep 2016;6:30713.

12. Yan X, Zhou Q, Vincent M, Deng Y, Yu J, Xu J, et al. Multifunctional biohybrid magnetite microrobots for imaging-guided therapy. Sci Robot 2017;2:eaaq1155.

13. Bozuyuk U, Yasa O, Yasa IC, Ceylan H, Kizilel S, Sitti M. Light-Triggered Drug Release from 3D-Printed Magnetic Chitosan Microswimmers. ACS Nano 2018;12:9617-25.

14. Peyer KE, Zhang L, Nelson BJ. Bio-inspired magnetic swimming microrobots for biomedical applications. Nanoscale 2013;5:1259-72.

15. Ornes S. Inner Workings: Medical microrobots have potential in surgery, therapy, imaging, and diagnostics. Proc Natl Acad Sci U S A 2017;114:12356-8.

16. Gilchrist RK, Medal R, Shorey WD, Hanselman RC, Parrott JC, Taylor CB. Selective inductive heating of lymph nodes. Ann Surg 1957;146:596-606.
17. Häfeli UO. Magnetically modulated therapeutic systems. Int J Pharm 2004;277:19-24.

18. Meyers PH, Cronic F, Nice CM Jr. Experimental approach in the use and magnetic control of metallic iron particles in the lymphatic and vascular system of dogs as a contrast and isotopic agent. Am J Roentgenol Radium Ther Nucl Med 1963;90:1068-77.

19. Lübbe AS, Bergemann C, Riess H, Schriever F, Reichardt P, Possinger K, et al. Clinical experiences with magnetic drug targeting: a phase I study with 4'-epidoxorubicin in 14 patients with advanced solid tumors. Cancer Res 1996;56:4686-93.

20. Hilal SK, Michelsen WJ, Driller J, Leonard E. Magnetically guided devices for vascular exploration and treatment. Radiology 1974;113:529-40.

21. Widder KJ, Senyei AE, Ranney DF. In vitro release of biologically active adriamycin by magnetically responsive albumin microspheres. Cancer Res 1980;40:3512-7.

22. Ghosh A, Fischer P. Controlled propulsion of artificial magnetic nanostructured propellers. Nano Lett 2009;9:2243-5.

23. Kummer MP, Abbott JJ, Kratochvil BE, Borer R, Sengul A, Nelson BJ. OctoMag: An Electromagnetic System for 5-DOF Wireless Micromanipulation. IEEE Transactions on Robotics 2010;26:1006-17.

24. Pirmoradi FN, Jackson JK, Burt HM, Chiao M. A magnetically controlled MEMS device for drug delivery: design, fabrication, and testing. Lab Chip 2011;11:3072-80.

25. Guo S, Sawamoto J, Pan Q. A novel type of microrobot for biomedical application. 2005 IEEE/ RSJ International Conference on Intelligent Robots and Systems. IEEE; 2005. p. 1047-52.

26. Guo S, Pan Q, Khamesee MB. Development of a novel type of microrobot for biomedical application. Microsystem Technologies 2008;14:307-14.

27. Dreyfus R, Baudry J, Roper ML, Fermigier M, Stone HA, Bibette J. Microscopic artificial swimmers. Nature 2005;437:862-5.

28. Bell DJ, Leutenegger S, Hammar KM, Dong LX, Nelson BJ. Flagella-like Propulsion for Microrobots Using a Nanocoil and a Rotating Electromagnetic Field, Proceedings 2007 IEEE International Conference on Robotics and Automation, Roma, 2007. p. 1128-33.

29. Zhang L, Abbott JJ, Dong L, Peyer KE, Kratochvil $\mathrm{BE}$, Zhang $\mathrm{H}$, et al. Characterizing the swimming properties of artificial bacterial flagella. Nano Lett 2009;9:3663-7.

30. Ludwig W. Physiol A-Neuroethol Sens Neural Behav Physiol. Comp J 1930;13:397-504.

31. Sanchez T, Welch D, Nicastro D, Dogic Z. Cilialike beating of active microtubule bundles. Science 2011;333:456-9.

32. Schenck JF. Safety of strong, static magnetic fields. J Magn Reson Imaging 2000;12:2-19. 
33. Zaremba LA. Guidance for industry and FDA staff: Criteria for significant risk investigations of magnetic resonance diagnostic devices. Center for Devices and Radiological Health; 2003.

34. Chen P, Güven S, Usta OB, Yarmush ML, Demirci U. Biotunable acoustic node assembly of organoids. Adv Healthc Mater 2015;4:1937-43.

35. Bouyer C, Chen P, Güven S, Demirtaş TT, Nieland TJ, Padilla F, et al. A Bio-Acoustic Levitational (BAL) Assembly Method for Engineering of Multilayered, 3D Brain-Like Constructs, Using Human Embryonic Stem Cell Derived Neuro-Progenitors. Adv Mater 2016;28:161-7.

36. Li S, Glynne-Jones P, Andriotis OG, Ching KY, Jonnalagadda US, Oreffo RO, et al. Application of an acoustofluidic perfusion bioreactor for cartilage tissue engineering. Lab Chip 2014;14:4475-85.

37. Feng J, Yuan J, Cho SK. Micropropulsion by an acoustic bubble for navigating microfluidic spaces. Lab Chip 2015;15:1554-62.

38. Ahmed D, Lu M, Nourhani A, Lammert PE, Stratton Z, Muddana HS, et al. Selectively manipulable acousticpowered microswimmers. Sci Rep 2015;5:9744.

39. Aghakhani A, Yasa O, Wrede P, Sitti M. Acoustically powered surface-slipping mobile microrobots. Proc Natl Acad Sci U S A 2020;117:3469-77.

40. Alapan Y, Yasa O, Yigit B, Yasa IC, Erkoc P, Sitti M. Microrobotics and microorganisms: Biohybrid autonomous cellular robots. Annual Review of Control, Robotics, and Autonomous Systems 2019;2: 205-30.

41. Martel S. Bacterial microsystems and microrobots. Biomed Microdevices 2012;14:1033-45.

42. Sowa Y, Rowe AD, Leake MC, Yakushi T, Homma M, Ishijima A, et al. Direct observation of steps in rotation of the bacterial flagellar motor. Nature 2005;437:916-9.

43. Wadhams GH, Armitage JP. Making sense of it all: bacterial chemotaxis. Nat Rev Mol Cell Biol 2004;5:1024-37.

44. Eisenbach M. Control of bacterial chemotaxis. Mol Microbiol 1996;20:903-10.

45. Pfennig N. Photosynthetic bacteria. Annu Rev Microbiol 1967;21:285-324.

46. Krulwich TA, Sachs G, Padan E. Molecular aspects of bacterial $\mathrm{pH}$ sensing and homeostasis. Nat Rev Microbiol 2011;9:330-43.

47. Kim D, Liu A, Diller E, Sitti M. Chemotactic steering of bacteria propelled microbeads. Biomed Microdevices 2012;14:1009-17.

48. Weibel DB, Garstecki P, Ryan D, DiLuzio WR, Mayer $\mathrm{M}$, Seto JE, et al. Microoxen: microorganisms to move microscale loads. Proc Natl Acad Sci U S A 2005;102:11963-7.

49. Zhuang J, Wright Carlsen R, Sitti M. pH-Taxis of Biohybrid Microsystems. Sci Rep 2015;5:11403.

50. Hosseinidoust Z, Mostaghaci B, Yasa O, Park BW, Singh AV, Sitti $M$. Bioengineered and biohybrid bacteria-based systems for drug delivery. Adv Drug Deliv Rev 2016;106:27-44.

51. Li D, Choi H, Cho S, Jeong S, Jin Z, Lee C, et al. A hybrid actuated microrobot using an electromagnetic field and flagellated bacteria for tumor-targeting therapy. Biotechnol Bioeng 2015;112:1623-31.

52. Jang D, Jeong J, Song H, Chung SK. Targeted drug delivery technology using untethered microrobots: a review. Journal of Micromechanics and Microengineering 2019;29:053002.

53. Ma X, Jannasch A, Albrecht UR, Hahn K, MiguelLópez A, Schäffer E, et al. Enzyme-Powered Hollow Mesoporous Janus Nanomotors. Nano Lett 2015;15:7043-50.

54. Ma X, Jang S, Popescu MN, Uspal WE, MiguelLópez A, Hahn K, et al. Reversed Janus Micro/ Nanomotors with Internal Chemical Engine. ACS Nano 2016;10:8751-9.

55. Paxton WF, Kistler KC, Olmeda CC, Sen A, St Angelo SK, Cao Y, et al. Catalytic nanomotors: autonomous movement of striped nanorods. J Am Chem Soc 2004;126:13424-31.

56. Paxton WF, Sen A, Mallouk TE. Motility of catalytic nanoparticles through self-generated forces. Chemistry 2005;11:6462-70.

57. Li J, Huang G, Ye M, Li M, Liu R, Mei Y. Dynamics of catalytic tubular microjet engines: dependence on geometry and chemical environment. Nanoscale 2011;3:5083-9.

58. Manjare M, Yang B, Zhao Y. Bubble-Propelled Microjets: Model and Experiment. The Journal of Physical Chemistry C 2013;117:4657-65.

59. Gibbs JG, Zhao YP. Autonomously motile catalytic nanomotors by bubble propulsion. Applied Physics Letters 2009;94:163104.

60. Xu T, Soto F, Gao W, Garcia-Gradilla V, Li J, Zhang $\mathrm{X}$, et al. Ultrasound-modulated bubble propulsion of chemically powered microengines. J Am Chem Soc 2014;136:8552-5.

61. Magdanz V, Stoychev G, Ionov L, Sanchez S, Schmidt OG. Stimuli-responsive microjets with reconfigurable shape. Angew Chem Int Ed Engl 2014;53:2673-7.

62. Wu Z, Li J, de Ávila BEF, Li T, Gao W, He Q, et al. Water-Powered Cell-Mimicking Janus Micromotor. Advanced Functional Materials 2015;25:7497-501.

63. Gao W, Dong R, Thamphiwatana S, Li J, Gao W, Zhang $\mathrm{L}$, et al. Artificial micromotors in the mouse's stomach: a step toward in vivo use of synthetic motors. ACS Nano 2015;9:117-23.

64. Solovev AA, Mei Y, Bermúdez Ureña E, Huang G, Schmidt OG. Catalytic microtubular jet engines self-propelled by accumulated gas bubbles. Small 2009;5:1688-92.

65. Gao W, Uygun A, Wang J. Hydrogen-bubble-propelled zinc-based microrockets in strongly acidic media. J Am Chem Soc 2012;134:897-900. 